\title{
Stabilization of Metastatic Uterine Leiomyosarcoma Using Pembrolizumab
}

\author{
Katherine Cotangco, DOㅜ; Mary Meram, DO2; and M. Patrick Lowe, $\mathrm{MD}^{3}$
}

\begin{abstract}
The estimated 5-year survival rate for patients with stage IV uterine leiomyosarcoma is $29 \%$, with a median survival of $<1$ year. This report describes a patient with stage IVB leiomyosarcoma who experienced stable disease for 15 months on pembrolizumab. A woman aged 62 years, gravida 2 para 2, with postmenopausal bleeding and a necrotic uterine fibroid underwent a dilation and curettage and was diagnosed with uterine leiomyosarcoma. CT imaging showed pulmonary and bony metastasis. She underwent surgical staging with biopsy-proved stage IVB disease. A mixed partial response was noted using first- and second-line chemotherapy. Molecular tumor profiling confirmed PD-L1 expression. The patient was started on pembrolizumab, and at the time of writing she is status post 23 cycles with stable disease. Pembrolizumab was well tolerated and aided prolonged disease stabilization.
\end{abstract}

J Natl Compr Canc Netw 2020;18(8):1012-1014 doi: $10.6004 /$ jnccn.2020.7562

${ }^{1}$ Department of Obstetrics and Gynecology, University of Illinois; ${ }^{2}$ Department of Obstetrics and Gynecology, Amita Health Hospital; and ${ }^{3}$ Gynecologic Cancer Institute, Chicago, Illinois.
Uterine leiomyosarcoma has an incidence of 0.64 per 100,000 patients. ${ }^{1}$ Although $60 \%$ of leiomyosarcomas are diagnosed at an early stage, they have high rates of recurrence and progression. ${ }^{1,2}$ Recurrence rates vary between $45 \%$ and $75 \%$, with median intervals to recurrence of 12 to 24 months. ${ }^{2}$ Uterine leiomyosarcoma spreads hematogenously and often metastasizes to the lungs. ${ }^{1}$ Estimated 5-year survival is stage-dependent and ranges from $76 \%$ in stage I disease to $29 \%$ in stage IV disease. ${ }^{3}$ Treatment of advanced-stage uterine leiomyosarcoma is systemic therapy with or without palliative therapy. ${ }^{4}$ There has been increasing interest in targeted cancer therapies. This report discusses the value of PD-L1 testing in a patient with stage IVB leiomyosarcoma with lung and bone metastasis who received third-line treatment using pembrolizumab and denosumab with stable disease for 15 months.

\section{Case Report}

A woman aged 62 years, gravida 2 para 2, presented with postmenopausal vaginal spotting and subsequently underwent a dilation and curettage. Pathology results suggested leiomyosarcoma. A CT scan of the chest, abdomen, and pelvis revealed a right uterine body peripherally enhancing partially calcified necrotic $5.8 \times$ $5.0-\mathrm{cm}$ mass, bilateral pulmonary nodules suspicious for metastatic disease, and possible bony vertebral metastasis. She underwent a total abdominal hysterectomy, bilateral salpingo-oophorectomy, and bilateral pelvic lymph node dissection for enlarged lymph nodes in June 2017. Final pathology results revealed leiomyosarcoma, negative for estrogen and progesterone receptors. Fine-needle aspiration of a single lung nodule confirmed metastatic disease. Bone biopsy of her vertebral lesion was deferred secondary to location. However, MRI of the spine confirmed metastatic lesions within the T12, L5, and S1 vertebral bodies and the right sacral alar. Thus, she was diagnosed as having stage IVB uterine leiomyosarcoma with histologically proved pulmonary metastasis and spinal metastasis per MRI.

A multidisciplinary decision was made to proceed with treatment involving intravenous gemcitabine at $900 \mathrm{mg} / \mathrm{m}^{2}$ and docetaxel at $35 \mathrm{mg} / \mathrm{m}^{2}$ plus pegfilgrastim every 21 days for a minimum of 6 cycles. ${ }^{5-8}$ The patient 
was also started on a 120-mg injection of denosumab every 28 days for bone metastasis. She was asymptomatic from her bony metastasis, and therefore palliative radiation was deferred. Repeat imaging after 3 cycles showed a mixed response of the lung and bone metastases, prompting molecular tumor profiling using immunohistochemistry and next-generation sequencing. Repeat CT imaging after cycle 6 showed a mixed response to treatment. Molecular tumor profiling using Caris Molecular Intelligence (Caris Life Sciences) confirmed a 90\% PD-L1 expression. The assay also confirmed estrogen and progesterone receptor negativity, pTEN positivity, and mismatch repair status stability (Table 1). After cycle 8 , the patient began experiencing dyspnea and fatigue. Her CT scans showed a mixed response with worsening pulmonary effusions. In light of her symptoms and the extent of the pulmonary disease, the decision was made to proceed with a chemotherapy regimen involving mesna $\left(2,500 \mathrm{mg} / \mathrm{m}^{2}\right)$, doxorubicin $\left(15 \mathrm{mg} / \mathrm{m}^{2}\right)$, ifosfamide $\left(2,000 \mathrm{mg} / \mathrm{m}^{2}\right)$, and dacarbazine $\left(250 \mathrm{mg} / \mathrm{m}^{2}\right)$ every 3 weeks while continuing denosumab. After 3 cycles, CT scans showed an interval decrease in pulmonary nodules and left pleural effusions and no change in her bony lesion. Repeat imaging after cycle 5 showed no change. Pembrolizumab was recommended given the patient's strong PD-L1 positivity, and she was started on intravenous pembrolizumab at $200 \mathrm{mg}$ every 21 days in June 2018. Imaging after 10 cycles showed stable disease. The patient experienced a mild dermatitis that resolved with topical steroids and developed grade 1 hypothyroidism before cycle 11 . She was started on levothyroxine at $25 \mathrm{mcg}$, and treatment resumed 2 weeks later. At the time of writing she is status post 23 cycles with stable disease noted on imaging.

\section{Table 1. Notable Biomarker Results}

\begin{tabular}{|lll|}
\hline Biomarker & Method & Result \\
\hline Total mutational load & \multicolumn{2}{l|}{} \\
\hline MSI & NGS & Intermediate | 8 mutations/mb \\
\hline Mismatch repair status & \multicolumn{2}{l|}{ Stable } \\
\hline PD-L1 & IHC & Proficient \\
\hline ERCC1 & IHC & Positive | 2+, 90\% \\
\hline TUBB3 & IHC & Positive | 2+, 75\% \\
\hline TS & IHC & Positive | 2+, 20\% \\
\hline RRM1 & IHC & Negative | 2+, $40 \%$ \\
\hline TOPO1 & IHC & Positive | 2+, 35\% \\
\hline ER & IHC & Negative | $1+, 5 \%$ \\
\hline PR & IHC & Negative | $0,100 \%$ \\
\hline TOP2A & IHC & Positive | 2+, 30\% \\
\hline
\end{tabular}

Abbreviations: ER, estrogen receptor; IHC, immunohistochemistry; mb, megabase; MSI, microsatellite instability; NGS, next-generation sequencing; $\mathrm{PR}$, progesterone receptor.

\section{Discussion}

The mainstay of treatment for advanced uterine leiomyosarcoma is systemic therapy with or without tumor-directed radiation therapy. Preferred regimens are single-agent doxorubicin or combination docetaxel/ gemcitabine. ${ }^{8}$ Although women treated with chemotherapy have improved survival, the prognosis is poor, with a median overall survival of $<2$ years. $^{2}$

Scientific advances in molecular testing have led to greater research in targeted cancer therapies, including immunotherapy. Checkpoint inhibitors such as nivolumab and pembrolizumab have gained increasing attention for the treatment of gynecologic malignancies. Pembrolizumab is a PD-1-blocking antibody that suppresses antitumor immunity. Detection of high PD-L1 expression may indicate which patients will benefit from targeting PD-1. In 2017, pembrolizumab was approved for the treatment of solid tumors with microsatellite instability or mismatch repair deficiencies. Next, the FDA approved pembrolizumab for the treatment of recurrent or metastatic cervical cancer with PD-L1 expression in 2018. In September 2019, pembrolizumab with lenvatinib was approved as a combined treatment of recurrent or metastatic endometrial cancer in patients previously treated with systemic therapy without microsatellite instability or mismatch repair deficiencies. $^{9}$

The role of pembrolizumab for the treatment of uterine leiomyosarcoma has not been extensively described in the literature. In 2017, Tawbi et al ${ }^{1}$ reported on a multicenter, 2-cohort, single-arm, open-label phase II trial examining the use of pembrolizumab in soft tissue and bone sarcoma in patients previously treated using up to 3 lines of chemotherapy $(\mathrm{N}=86)$. A subgroup analysis of uterine leiomyosarcoma $(\mathrm{N}=10)$ showed no objective response to pembrolizumab. No serious adverse events were reported. In addition, PD-L1 expression was explored as a potential marker for clinical benefit; however, none of the leiomyosarcoma tumors expressed PD-L1. George et al published a case report in 2017 describing a positive response to pembrolizumab after primary resection in a patient with recurrent metastatic uterine leiomyosarcoma with $<5 \%$ PD-L1 expression ${ }^{10}$; she remained in remission on pembrolizumab monotherapy for $>24$ months.

The extent of PD-Ll expression may be clinically predictive in patients who may benefit from PD-1 blockade. The KEYNOTE-028, a multicohort, open-label phase Ib basket trial, investigated the efficacy of pembrolizumab in PD-L1-positive tumors. ${ }^{11}$ In the endometrial cancer subgroup analysis $(\mathrm{N}=75), 48 \%$ of patients expressed PD-L1 with an objective response rate of $13.5 \%$. This result was comparable to existing chemotherapy regimens. The investigators concluded that PD-L1 expression was indicative of the predicted response to PD-1 blockade. 
The patient described in this report had a $>90 \%$ PD-L1 expression, and therefore expanded access for pembrolizumab was approved.

\section{Conclusions}

At the time of writing, our patient has experienced stable disease, with minimal adverse effects, over 23 cycles of pembrolizumab and denosumab. PD-L1 expression may be a clinical biomarker for patients with endometrial cancer. Although the SARC028 trial $^{1}$ found no benefit with pembrolizumab in the treatment of leiomyosarcoma, none of the tumors expressed PD-L1. Given the paucity of data on immunotherapy for the treatment of metastatic leiomyosarcoma, it is difficult to extrapolate literature from other disease sites to our unique patient. Pembrolizumab in our patient was welltolerated, aided in prolonged disease stabilization, and will be continued until disease progression or unacceptable toxicity. Clinicians should consider PD-L1 testing in patients with metastatic leiomyosarcoma.

Submitted November 6, 2019; accepted for publication March 19, 2020.

Disclosures: The authors have disclosed that they have not received any financial consideration from any person or organization to support the preparation, analysis, results, or discussion of this article.

Correspondence: Katherine Cotangco, DO, Department of Obstetrics and Gynecology, University of Illinois, 1801 West Taylor Street, Suite 1E, Chicago, IL 60612. Email: krcotangco@gmail.com

\section{References}

1. Tawbi HA, Burgess $M$, Bolejack $V$, et al. Pembrolizumab in advanced soft-tissue sarcoma and bone sarcoma (SARC028): a multicentre, twocohort, single-arm, open-label, phase 2 trial. Lancet Oncol 2017;18: 1493-1501.

2. Roberts ME, Aynardi JT, Chu CS. Uterine leiomyosarcoma: a review of the literature and update on management options. Gynecol Oncol 2018;151: 562-572.

3. Hensley ML, Barrette BA, Baumann K, et al. Gynecologic Cancer InterGroup (GCIG) consensus review: uterine and ovarian leiomyosarcomas. Int J Gynecol Cancer 2014;24(Suppl 3):S61-66.

4. Abu-Rustum NR, Yashar CM, Bean S, et al. NCCN Clinical Practice Guidelines in Oncology: Uterine Neoplasms. Version 1.2020. Accessed June 30, 2020. To view the most recent version, visit NCCN.org

5. Hensley ML, Blessing JA, Degeest $K$, et al. Fixed-dose rate gemcitabine plus docetaxel as second-line therapy for metastatic uterine leiomyosarcoma: a Gynecologic Oncology Group phase II study. Gynecol Oncol 2008;109:323-328.

6. Hensley ML, Blessing JA, Mannel R, et al. Fixed-dose rate gemcitabine plus docetaxel as first-line therapy for metastatic uterine leiomyosarcoma: a Gynecologic Oncology Group phase II trial. Gynecol Oncol 2008;109: 329-334.

7. Hyman DM, Grisham RN, Hensley ML. Management of advanced uterine leiomyosarcoma. Curr Opin Oncol 2014;26:422-427.

8. Lee HY, Shin SJ, Kim HS, et al. Weekly gemcitabine and docetaxel in refractory soft tissue sarcoma: a retrospective analysis. Cancer Res Treat 2012;44:43-49.

9. Stewart J. Keytruda approval history. Accessed March 25, 2020. Available at: https://www.drugs.com/history/keytruda.html

10. George S, Miao D, Demetri GD, et al. Loss of PTEN is associated with resistance to anti-PD-1 checkpoint blockade therapy in metastatic uterine leiomyosarcoma. Immunity 2017;46:197-204.

11. Ott PA, Bang YJ, Berton-Rigaud D, et al. Safety and antitumor activity of pembrolizumab in advanced programmed death ligand 1-positive endometrial cancer: results from the KEYNOTE-028 study. J Clin Oncol 2017;35:2535-2541. 